Rebecca Spain, MD, $\mathrm{MSPH}$

Katherine Powers, BA, CCRP

Charles Murchison, MS

Elizabeth Heriza, MD

Kimberly Winges, MD

Vijayshree Yadav, MD, MSCR

Michelle Cameron, MD, PT

Ed Kim, MD

Fay Horak, PhD, PT Jack Simon, MD, PhD

Dennis Bourdette, MD

Correspondence to

Dr. Spain:

spainr@ohsu.edu

\section{Lipoic acid in secondary progressive MS}

\section{A randomized controlled pilot trial}

\section{OPEN}

\section{ABSTRACT}

Objective: To determine whether lipoic acid (LA), an endogenously produced antioxidant, slowed the whole-brain atrophy rate and was safe in secondary progressive MS (SPMS).

Methods: Patients with SPMS aged 40-70 years enrolled in a single center, 2-year, double-blind, randomized trial of daily oral 1,200 mg LA vs placebo. Primary outcome was change in annualized percent change brain volume (PCBV). Secondary outcomes were changes in rates of atrophy of segmented brain, spinal cord, and retinal substructures, disability, quality of life, and safety. Intention-to-treat analysis used linear mixed models.

Results: Participation occurred between May 2, 2011, and August 14, 2015. Study arms of LA $(n=27)$ and placebo $(n=24)$ were matched with mean age of 58.5 (SD 5.9) years, $61 \%$ women, mean disease duration of 29.6 (SD 9.5) years, and median Expanded Disability Status Score of 6.0 (interquartile range 1.75). After 2 years, the annualized PCBV was significantly less in the LA arm compared with placebo ( -0.21 [standard error of the coefficient estimate (SEE) 0.14] vs -0.65 [SEE 0.10], 95\% confidence interval [CI] 0.157-0.727, $p=0.002$ ). Improved Timed 25Foot Walk was almost but not significantly better in the LA than in the control group $(-0.535$ [SEE 0.358] vs 0.137 [SEE 0.247], $95 \% \mathrm{Cl}-1.37$ to $0.03, p=0.06$ ). Significantly more gastrointestinal upset and fewer falls occurred in LA patients. Unexpected renal failure $(n=1)$ and glomerulonephritis $(n=1)$ occurred in the LA cohort. Compliance, measured by pill counts, was $87 \%$.

Conclusions: LA demonstrated a $68 \%$ reduction in annualized PCBV and suggested a clinical benefit in SPMS while maintaining favorable safety, tolerability, and compliance over 2 years.

ClinicalTrials.gov identifier: NCT01188811.

Classification of evidence: This study provides Class I evidence that for patients with SPMS, LA reduces the rate of brain atrophy. Neurol Neuroimmunol Neuroinflamm 2017;4:e374; doi: 10.1212/ NXI.0000000000000374

\section{GLOSSARY}

$\mathbf{A E}=$ adverse event; $\mathbf{C l}=$ confidence interval; $\mathbf{E A E}=$ experimental autoimmune encephalomyelitis; $\mathbf{E D S S}=$ Expanded Disability Status Scale; GI = gastrointestinal; ITT = intention to treat; $\mathbf{L A}=$ lipoic acid; $\mathbf{M P}-\mathbf{R A G E}=$ magnetization-prepared rapid acquisition gradient echo; $\mathbf{O C T}=$ optical coherence tomography; $\mathbf{P C B V}=$ percent change brain volume; $\mathbf{P I}=$ principal investigator; RRMS = relapsing-remitting MS; SAE = serious $A E ;$ SEE = standard errors of the coefficient estimate; SPMS = secondary progressive MS.

By 2 decades, the majority with relapsing-remitting MS (RRMS) have secondary progressive MS (SPMS). SPMS pathophysiology likely involves mitochondrial dysfunction, microglial activation, vascular endothelial disruption, and effects of meningeal lymphoid-like tissues. ${ }^{1}$ The resulting neurodegeneration and accelerated brain atrophy correlate with functional disability; thus whole-brain atrophy is the current gold-standard MRI surrogate outcome measure for
Supplemental data at Neurology.org/nn
From the Neurology Division (R.S., V.Y., M.C., E.K., D.B.), Research Service (K.P., E.H.), and Department of Ophthalmology (K.W.), Veterans Affairs Portland Health Care System, OR; and Department of Neurology (R.S., C.M., K.W., V.Y., E.K., F.H., J.S., D.B.), Advanced Imaging Research Center (K.P.), and Casey Eye Institute (K.W.), Oregon Health \& Science University, Portland.

Funding information and disclosures are provided at the end of the article. Go to Neurology.org/nn for full disclosure forms. The Article Processing Charge was funded by the authors.

This is an open access article distributed under the terms of the Creative Commons Attribution-NonCommercial-NoDerivatives License 4.0 (CC BY-NC-ND), which permits downloading and sharing the work provided it is properly cited. The work cannot be changed in any way or used commercially without permission from the journal. 
SPMS trials. ${ }^{2}$ Targeting specific pathophysiologic processes is a rational strategy for treating SPMS.

Lipoic acid (LA) is an endogenously produced antioxidant with multiple biological functions including free-radical scavenging, metallic ion chelation, regeneration of intracellular glutathione, and oxidative damage repair of macromolecules. ${ }^{3}$ In mitochondria, the LA/dihydrolipoic acid redox couple is a key cofactor for the pyruvate dehydrogenase complex of oxidative respiration and aids nucleic acid synthesis. ${ }^{4}$ LA modulates the PKB/ Akt signaling pathway important for vascular endothelial integrity, affects transcription factor Nrf2, and acts as an insulin mimetic., ${ }^{5,6}$

Our center and others have shown that LA reduced disability in experimental autoimmune encephalomyelitis (EAE), diminished inflammatory cell migration into spinal cords and optic nerves, and inhibited macrophage/ microglial activation. ${ }^{7,8}$ Oral ingestion of LA by patients with MS produced blood levels comparable with those in EAE. ' In clinical trials, LA is well tolerated; common adverse reactions were gastrointestinal (GI) intolerance, headache, malodorous urine, and rash. ${ }^{10,11}$ Herein, we report results of a 2year, randomized controlled trial to determine whether LA reduced rates of whole-brain atrophy, slowed clinical deterioration, and was safe in SPMS.

METHODS Study design. This was a prospective, single-site, 2-year, phase II, double-blind, randomized, placebo-controlled trial of $1,200 \mathrm{mg}$ daily oral racemic LA to answer the following primary research question with Class I level of evidence: would LA reduce the rate of whole-brain atrophy in SPMS? Secondary research questions were to determine whether LA would reduce rates of atrophy of segmented brain, spinal cord, and retinal substructures, reduce deterioration of disability and quality of life, and be safe in SPMS. Recruitment occurred between May 2011 and October 2013, with last visit being in August 2015. The study was conducted at the Veterans Affairs Portland Health Care System (VAPORHCS), Portland, OR, with some procedures at Oregon Health \& Science University (OHSU), Portland, OR.

Participants. Inclusion criteria were ages $40-70$ years, prior RRMS (2005 McDonald criteria), and current SPMS defined by MS disability progression in the absence of clinical relapse during the prior 5 years as determined by the principal investigator (PI) based on history and chart review. ${ }^{12}$ Progression was defined as sufficient to change a Functional System on the Expanded Disability Status Scale (EDSS) or effect a meaningful functional change (e.g., stopped working due to cognitive decline).$^{13}$ Participants were permitted to start, stop, or continue glatiramer acetate or $\beta$-interferon during the study. Exclusion criteria were use of natalizumab, immunosuppressants, chemotherapies, or scheduled IV corticosteroid treatments within 1 year of enrollment, corticosteroid treatment for relapse within 60 days of enrollment, LA within 30 days of enrollment, MRI constraints, self-reported ocular disease that could confound optical coherence tomography (OCT) interpretation, pregnant or breastfeeding, significant active concurrent illness, uncontrolled or insulindependent diabetes, and lack of English fluency. Because of slow recruitment during the first 8 months, the EDSS limit of 6.0 was eliminated.

Standard protocol approvals, registrations, and patient consents. The study was approved by VAPORHCS and OHSU Institutional Review Boards. Written consent was obtained from all the participants. The study was registered on ClinicalTrials.gov (NCT01188811) and conducted following 2010 CONSORT guidelines. ${ }^{14}$

Study personnel roles. The PI conducted screening visits and initial EDSS examinations for randomization, evaluated adverse events (AEs), and served as the study monitor. Blinded neurologists and a neurology advanced practitioner served as EDSS examiners. Blinded study coordinators collected mobility data, questionnaires, and maintained databases.

Investigational drug. An Investigational New Drug indication was obtained by the PI (no. 110132). Pure Encapsulations (Sudbury, MA) provided gelatin capsules containing $600 \mathrm{mg}$ racemic LA or placebo. Placebo capsules contained Avicel (microcellulose crystal) and $4.3 \mathrm{mg}$ of quercetin (a bioflavonoid) that rendered the placebo a yellow color, similar to LA. Expiration of the study drug was extended once during the study by Pure Encapsulations after retesting of sample capsules determined continued stability.

Study timeline. Screen and baseline visits were $\leq 30$ days apart. Subsequent visits at months $3,6,12,18$, and 24 occurred \pm 2 weeks. A month was defined as 4 weeks for scheduling purposes. MRI and OCT occurred at baseline, months 12 and 24 . Clinical outcome measures were collected at baseline and every 6 months. Safety laboratory measures were performed at each visit. Telephone calls occurred between visits and after study completion to capture AEs.

Outcomes. Primary outcome was difference in annual percent change brain volume (PCBV) on MRI by Structural Image Evaluation, using Normalisation, of Atrophy (SIENA). Secondary outcomes included atrophy rates of segmented brain, spinal cord, and retinal substructures, changes in disability, quality of life, and safety.

MRI acquisition protocol. MRI acquisition and analyses details are shown in appendix e-1 at Neurology.org/nn. The following sequences were acquired using a Philips Achieva 3.0T X-series with Quasar Dual gradient systems: (1) 3D highresolution magnetization-prepared rapid acquisition gradient echo (3D MP-RAGE) with $1 \mathrm{~mm}^{3}$ voxels for high-resolution structural (T1-weighted) information. The upper cervical spinal cord was intentionally included in the series through positioning; (2) 3D fluid-attenuated inversion recovery (3D FLAIR) series with $1 \mathrm{~mm}^{3}$ voxels; (3) conventional $3 \mathrm{~mm}$ (0.3 gap) axial 2D proton density/T2-weighted sequences with in-plane resolution $1 \mathrm{~mm}^{2}$; and (4) 3-mm sagittal 2D proton density/T2-weighted spinal cord sequences. No intravascular contrast was used.

MRI analyses. MRIs were reviewed by a neuroradiologist for unexpected findings. A single trained MRI analyst performed lesion counts, volumetrics, and cortical thickness analyses. The 


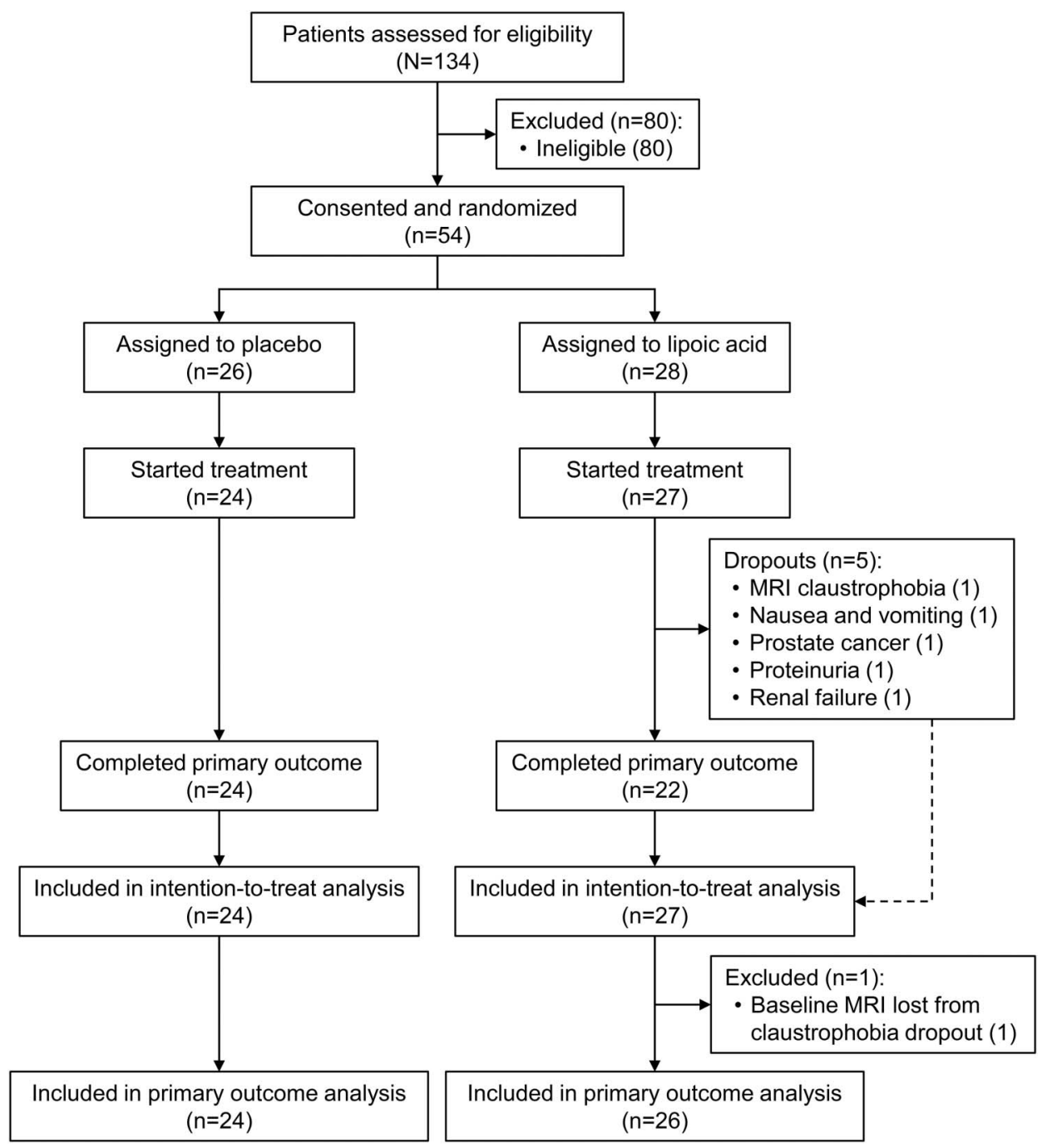

PI conducted spinal cord cross-sectional thickness and lesion occupancy analyses. Both were directed by the study neuroradiologist. Cerebral T2-hyperintense lesion volumes and maps were obtained using Lesion TOpology-preserving Anatomical Segmentation (Lesion-TOADS). ${ }^{15}$ FSL tools were used to create lesion-filled MP-RAGE images. SIENAX was used to determine cross-sectional whole-brain, white, and grey matter volumes. ${ }^{16}$ Whole-brain atrophy was determined using SIENA from the FSL package. ${ }^{16}$ Subcortical deep grey matter volumes were measured using FIRST. ${ }^{16}$ Cortical thickness, cortical volumetric segmentation, and analyses of these were performed with FreeSurfer (surfer.nmr.mgh.harvard.edu/) and its longitudinal processing stream. ${ }^{17}$ Spinal cord cross-sectional area was recorded at C1. An in-house estimation of the relative percent of the spinal cord from the foramen magnum to the lower edge of C7 occupied by MS lesions was devised for descriptive purposes. MRIs were graded for quality (good, fair, poor, and unusable). Poor and unusable scans were excluded from the analyses.

OCT. Participants underwent spectral domain OCT (Cirrus HDOCT; Carl Zeiss Meditec, Inc., Dublin, CA) in each eye after pharmacologic dilation ( $1 \%$ tropicamide and $0.5 \%$ proparacaine hydrochloride). Peripapillary and macular scans were obtained with Optic Disc Cube $200 \times 200$ and Macular Cube $512 \times 128$ protocols, respectively. OCTs were reviewed by an experienced neuro-ophthalmologist. Excluded scans had confounding findings, artifact, misalignment, or signal strength less than 7 .

Clinical measures. Disability was captured by the EDSS. The same EDSS examiner was used for a given participant throughout the study to the extent possible. Mobility measures for ambulatory participants were Timed 25-Foot Walk (T25FW), Multiple Sclerosis Walking Scale (MSWS-12) Questionnaire, and Activities-specific Balance Confidence (ABC) Questionnaire. ${ }^{18-20}$ Symbol Digit Modalities Test (SDMT) tested cognition. ${ }^{21}$ RAND 36-Item Short Form Health Survey assessed quality of life. $^{22}$

Safety monitoring. AEs were categorized using the Common Terminology Criteria for Adverse Events version 4.0 (CTCAE v4.0). Unscheduled visits occurred for relapses, AEs, and early study termination visits. Safety monitoring laboratory tests (complete blood count and kidney and liver panels) were checked at each visit. The Columbia-Suicide Severity Rating Scale (C-SSRS) was administered at every visit. A 3-member data safety and monitoring board met every year.

Sample size, randomization, and blinding. The study was powered for comparison of the primary outcome, PCBV, as 
estimated by Altmann et al. ${ }^{23}$ using an SD of 1.51 for SIENA atrophy, 2-year study length with yearly MRIs, and 60\% effect size. A sample size of 23 per arm was needed to obtain $80 \%$ power and a significance of $p<0.05$. Projected enrollment was increased to allow for dropouts. Participants were assigned to LA or placebo in a 1:1 manner by the unblinded research pharmacist following a permutated block randomization based on EDSS $\leq 4.5$ or $>4.5 .{ }^{13}$ All other study personnel were blinded to the treatment assignment. MRIs were labeled with additional randomly generated numbers during analyses to further reduce the risk of bias. Statistical analysis was performed by a blinded statistician for primary, secondary, and safety outcome measures.

Statistical methods. Intention-to-treat (ITT) analysis used linear mixed models to evaluate the effect of LA on annualized PCBV. Mixed models were used to adjust for withinparticipant serial correlation, to account for the repeated measurements of the longitudinal design, and to include all study participants. Models were corrected for participant age, sex, and MS duration with standard model diagnostics to identify overly influential leverage points. Multiple comparisons were accounted for using the Holm-Sidak correction within the study outcome domains. Outlier data points were identified through standard diagnostic techniques using combinations of data point leverage, individual residuals, and Cook distance to identify overly influential observations and exclude them from baseline and 2-year change analyses. Data from participants taking a reduced dose of LA $(\mathrm{n}=2)$ were not handled differently in outcome analyses as their limited number made subgroup assessment intractable. Mixedmodel results are reported as rates of change with variance represented by the standard errors of the coefficient estimates (SEEs). Post hoc analysis of the primary outcome measure was conducted adding the baseline whole-brain volume and baseline T2-lesion volume as covariates to the model. All analyses were performed using R 3.3.1 with additional utility from the lme4 package. ${ }^{24,25}$

RESULTS Of the 54 consented and randomized, 51 participants (27 LA and 24 placebo) took at least 1 dose of study drug and were included in the ITT analyses (figure 1). Forty-six participants completed the study (22 LA and 24 placebo). The 5 dropouts in the
LA cohort were for reasons of claustrophobia during MRI, prolonged nausea and vomiting which resolved on cessation of LA, and significant concurrent illness (prostate cancer, proteinuria, and worsening renal function). Because the dropout with claustrophobia did not complete the baseline MRI, the LA cohort ITT sample size for PCBV was 26 (figure 1).

Baseline demographics are presented in table 1. The LA and control cohorts were overall matched for age, sex, MS duration, education, and disability. There were no significant differences between treatment arms (all $p>0.05$ ). Table 2 presents the baseline values of the main study outcome measures by study arms. The LA cohort had a significantly larger baseline whole-brain $(p=0.004)$ and total deep grey matter volumes $(p=0.042)$. Controls had larger baseline normalized T2-lesion volumes.

Compliance with study drug was $87 \%$ by pill counts. Two participants took a halved dose of LA per protocol for the majority of the study, one each for gastritis and elevated alkaline phosphatase levels.

Rate of brain atrophy and secondary imaging outcomes. After 2 years, participants taking LA had significantly less annualized PCBV $(-0.21 \%$ [SEE 0.14]) than controls $(-0.65 \%$ [SEE 0.10], $p=0.002)$, with the beneficial effect size of LA treatment observed to be a $0.44 \% \pm 0.29 \%$ improvement in the rate of whole-brain atrophy $(95 \%$ confidence interval [CI] 0.157-0.727). This change corresponds to a $68 \%$ reduction in the rate of brain atrophy in LA vs placebo (figure 2A, table 3). In post hoc analysis, baseline whole-brain volume was not associated with any of the other controlling covariates (age, sex, and disease duration), nor did it affect the differences in PCBV between the study cohorts. Likewise, T2-

Table 1 Baseline demographics and clinical characteristics of study participants by treatment $(n=51)$

\begin{tabular}{|c|c|c|c|c|}
\hline & \multicolumn{2}{|c|}{$\mathrm{LA}(\mathrm{n}=27)$} & \multicolumn{2}{|c|}{ Placebo $(n=24)$} \\
\hline & $\begin{array}{l}\text { No. or } \\
\text { median }\end{array}$ & $\begin{array}{l}\text { SD, \%, or IQR } \\
\text { (range) }\end{array}$ & $\begin{array}{l}\text { No. or } \\
\text { median }\end{array}$ & $\begin{array}{l}\text { SD, \%, or IQR } \\
\text { (range) }\end{array}$ \\
\hline Age, y & 57.9 & 6.7 & 59.7 & 6.0 \\
\hline Women & 16 & 59 & 15 & 63 \\
\hline MS duration, y & 30.9 & 9.3 & 29.1 & 9.9 \\
\hline Education > high school & 25 & 93 & 21 & 88 \\
\hline EDSS & 5.5 & $2.5(3.0-8.0)$ & 6 & $1.5(3.0-9.0)$ \\
\hline EDSS $\leq 4.5$ & 11 & 40.8 & 9 & 37.5 \\
\hline Taking disease-modifying therapy ${ }^{a}$ & 12 & 44 & 11 & 46 \\
\hline
\end{tabular}

Abbreviations: EDSS = Expanded Disability Status Scale; IQR = interquartile range; $L A=$ lipoic acid.

${ }^{a}$ LA: glatiramer acetate $(n=6)$, interferon- $\beta$ 1a $(n=4)$, interferon- $\beta$ 1b $(n=2)$; placebo: glatiramer acetate $(n=5)$, interferon- $\beta 1 \mathrm{a}(\mathrm{n}=3)$, interferon- $\beta 1 \mathrm{~b}(\mathrm{n}=3)$. 


\begin{tabular}{|c|c|c|c|c|c|}
\hline \multirow[t]{3}{*}{ Baseline differences betw } & \multirow[b]{3}{*}{$\begin{array}{l}\text { Sample sizea } \\
\text { LA, placebo }\end{array}$} & \multirow{2}{*}{\multicolumn{2}{|c|}{ ome measures }} & \multirow{2}{*}{\multicolumn{2}{|c|}{ (mean, SD) }} \\
\hline & & & & & \\
\hline & & No. & SD & No. & SD \\
\hline Normalized whole-brain volume, cubic $\mathrm{cm}^{\mathrm{b}}$ & 26,24 & 1,451 & 63 & 1,395 & 66 \\
\hline Total deep grey matter volume, cubic $\mathrm{cm}^{\mathrm{c}}$ & 26,24 & 806.4 & 7.9 & 764.6 & 6.6 \\
\hline Cortical thickness, $\mathrm{mm}^{\mathrm{d}}$ & 23,21 & 2.3 & 0.1 & 2.3 & 0.1 \\
\hline Normalized T2-lesion volume, cubic $\mathrm{cm}^{\mathbf{e}}$ & 20,24 & 10.0 & 7.3 & 14.9 & 8.3 \\
\hline $\mathrm{C} 1$ cross-sectional area, squared $\mathrm{mm}^{\mathrm{f}}$ & 22,23 & 68.4 & 11.0 & 68.2 & 9.9 \\
\hline $\begin{array}{l}\text { Cervical spinal cord lesion occupancy, } \\
\text { cubic cm }\end{array}$ & 24,23 & 8.8 & 6.4 & 11.0 & 6.7 \\
\hline Average RNFL thickness, $\mu \mathrm{m}$ & 42,38 eyes & 78.2 & 9.8 & 76.3 & 10.8 \\
\hline Average GCIPL thickness, $\mu \mathrm{m}$ & 38,36 eyes & 68.8 & 8.9 & 65.9 & 8.9 \\
\hline SDMT, number correct & 27,24 & 39.7 & 11.2 & 39.1 & 8.9 \\
\hline Timed 25 Foot Walk, s & 23,19 & 11.7 & 11.3 & 11.5 & 9.6 \\
\hline
\end{tabular}

Abbreviations: GCIPL = retinal ganglion cell plus inner plexiform layer; $\mathrm{LA}=$ lipoic acid; $\mathrm{RNFL}=$ retinal nerve fiber layer; SDMT $=$ Symbol Digit Modalities Test.

a Sample sizes reflect removal of outliers and for exclusion reasons specified in Methods.

bSIENAX.

${ }^{c}$ FIRST FSL.

${ }^{\mathrm{d}}$ FreeSurfer.

${ }^{e}$ MIPAV, Lesion-TOADS, semiautomated segmentation.

${ }^{f}$ MIPAV.

lesion volume change over the study period was not associated with any of the correcting covariates, including baseline T2-lesion volume. Individual 2-year changes in PCBV for LA $(-0.45 \%$ [SEE $0.71])$ vs controls $(-1.31 \%$ [SEE 1.10$], p=0.001)$ in study completers are shown (figure 2B). Brain segmentation and OCT did not reveal significant differences in annualized rates of change between study arms; the increase in T2-lesion volume in the LA cohort was almost but not significantly different from controls (414.6 [SEE 201.2] vs 33.1 [SEE 134.7], $p=0.058$, table 3 ).

Clinical outcomes. Among clinical outcomes, the LA cohort demonstrated an improvement in the T25FW that was almost but not significantly different from controls $(-0.535$ [SEE 0.358] vs 0.137 [SEE 0.247], 95\% CI -1.37 to $0.03, p=0.060$ ).

AEs. AEs are presented in table e-1. Numbers of AEs were similar between LA and controls (80 and 68, respectively, $p=0.87$ ), with 6 serious AEs (SAEs) each. More GI upset (14 [SD 17\%] vs 2 [SD 3\%], $p=0.007)$ and fewer falls (12 [SD 15\%] vs 26 [SD $38 \%], p=0.03$ ) occurred in the LA cohort. One LA participant developed a nonbothersome vesicular rash, similar to a previously reported one, that resolved within 6 weeks of study completion. ${ }^{11}$ One relapse occurred in each study cohort, neither affecting the subsequent EDSS examination. There was no new suicidality by C-SSRS, and no differences between cohorts in new laboratory abnormalities;

Figure 2 Differences in brain atrophy at 2 years between LA and control cohorts
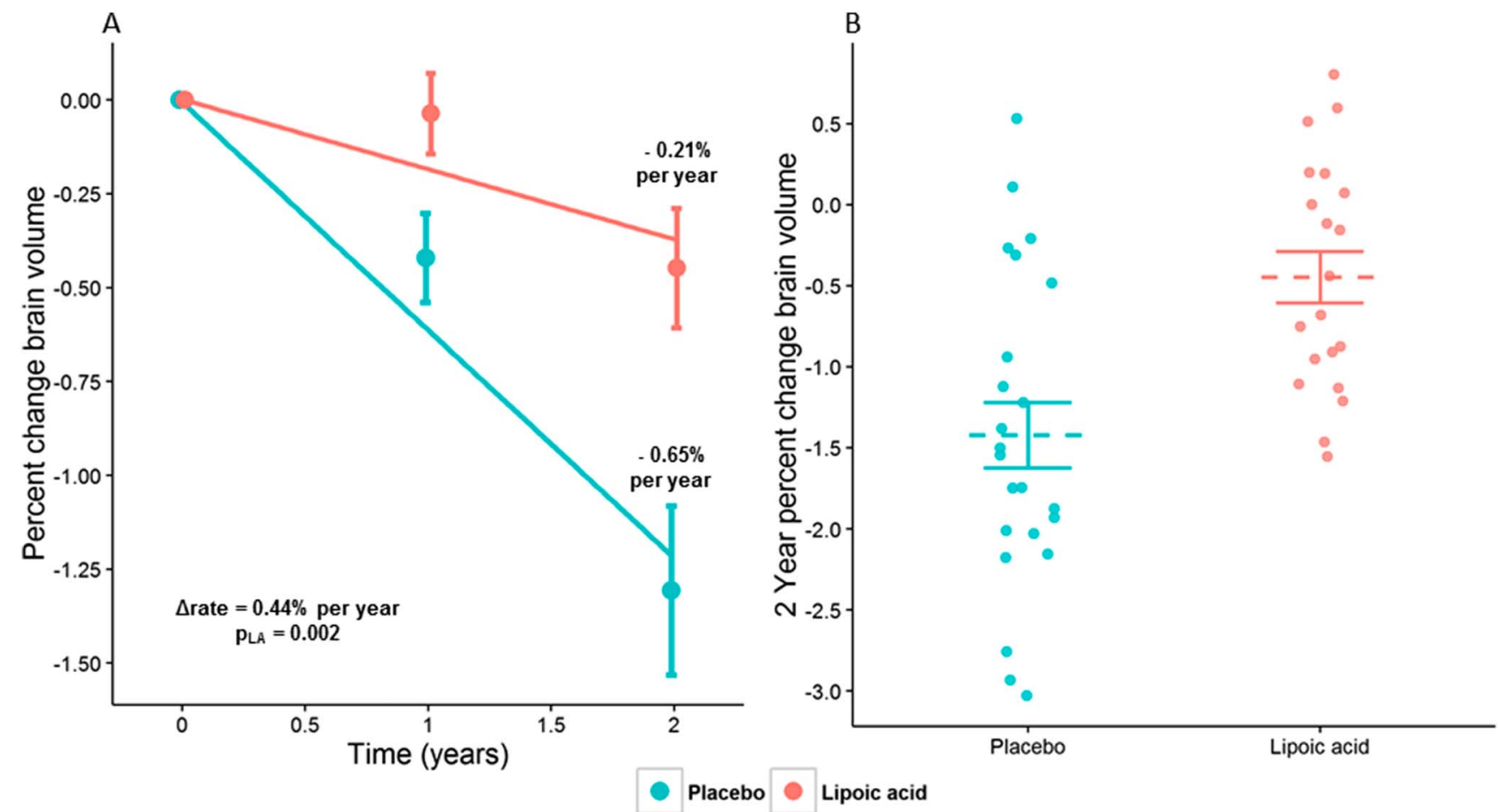

Annualized percent change brain volume (PCBV) between LA and placebo cohorts using intention-to-treat analysis of 51 participants with secondary progressive MS (A). Two-year PCBV from study completers is shown and demonstrates significantly less PCBV in the LA cohort ( $n=22,-0.45 \%$ [SEE 0.71 ]) than controls $(n=24,-1.31 \%$ [SEE 1.10], $p=0.001, B) . L A=$ lipoic acid; SEE = standard errors of the coefficient estimate. 


\begin{tabular}{|c|c|c|c|c|c|}
\hline \multicolumn{6}{|l|}{ Table $3 \quad$ Study outcomes } \\
\hline & \multicolumn{2}{|l|}{ LA } & \multicolumn{2}{|l|}{ Placebo } & \multirow[b]{2}{*}{$p$ Value } \\
\hline & No. & SEE & Number & SEE & \\
\hline Percent change brain volume ${ }^{a}$ & -0.214 & 0.143 & -0.653 & 0.097 & 0.002 \\
\hline Total deep grey matter volume, cubic $\mathbf{m m}^{\mathrm{b}}$ & 42.0 & 268.1 & -265.2 & 185.5 & 0.25 \\
\hline Cortical thickness percent change $^{c}$ & -0.137 & 0.339 & -0.158 & 0.24 & 0.95 \\
\hline T2-lesion volume, cubic $\mathrm{cm}^{\mathrm{d}}$ & 414.6 & 201.2 & 33.1 & 134.7 & 0.058 \\
\hline C1 cross-sectional area, squared $\mathrm{mm}^{\mathrm{e}}$ & -0.113 & 0.774 & -0.984 & 0.52 & 0.26 \\
\hline Average RNFL, $\mu \mathrm{m}$ & -0.279 & 0.744 & -0.286 & 0.518 & 0.99 \\
\hline Average GCIPL, $\mu \mathrm{m}$ & -0.322 & 0.595 & -0.145 & 0.417 & 0.77 \\
\hline Timed 25-Foot Walk, s & -0.535 & 0.358 & 0.137 & 0.247 & 0.060 \\
\hline$A B C$, units & 2.51 & 1.679 & 0.177 & 1.174 & 0.16 \\
\hline MSWS-12, units & 2.49 & 1.36 & 2.99 & 0.95 & 0.71 \\
\hline RAND, physical, units & 0.589 & 0.876 & -0.667 & 0.61 & 0.15 \\
\hline RAND, mental, units & -0.385 & 1.187 & 0.522 & 0.827 & 0.45 \\
\hline SDMT, units & 2.09 & 0.93 & 2.59 & 0.64 & 0.59 \\
\hline EDSS stable or improved & $61 \%$ & - & $71 \%$ & - & OR $1.33, p=0.77$ \\
\hline Taking disease-modifying therapy ${ }^{f}$ & 11 & $5 \%$ & 11 & $46 \%$ & - \\
\hline
\end{tabular}

Abbreviations: $A B C=$ Activities of Balance Confidence; EDSS $=$ Expanded Disability Status Scale; GCIPL $=$ retinal ganglion cell plus inner plexiform layer; LA = lipoic acid; MSWS-12 = Multiple Sclerosis Walking Scale 12; OR = odds ratio; RAND = RAND 36-Item Short Form Health Survey; RNFL = retinal nerve fiber layer; SEE = standard errors of the coefficient estimate; SDMT $=$ Symbol Digit Modalities Test.

Annualized rates of change (SD) for MRI and clinical outcomes taken from intention-to-treat mixed models across the 2year study period. Significant differences are indicated in italics. Rates of change, SEEs, and $p$ values taken from model corrected outputs.

a SIENAX.

${ }^{\mathrm{b}}$ FIRST FSL.

${ }^{\mathrm{c}}$ FreeSurfer.

${ }^{\mathrm{d}}$ MIPAV, Lesion-TOADS, semiautomated segmentation.

${ }^{e}$ MIPAV.

${ }^{f}$ LA cohort: dimethyl fumarate $(n=1)$, glatiramer acetate $(n=5)$, interferon- $\beta$ 1a $(n=3)$, interferon- $\beta$ 1b $(n=2)$; placebo cohort: glatiramer acetate $(n=5)$, interferon- $\beta 1 a(n=3)$, interferon- $\beta 1 b(n=3)$.

however, only those taking LA required a dose reduction per protocol $(\mathrm{n}=2)$. The SAE thought directly related to LA was vomiting and dehydration requiring hospitalization which resolved once stopping LA. Notable AEs leading to dropout were one LA patient with baseline elevated creatinine progressing to renal failure and another with proteinuria due to glomerulonephritis. A consulting nephrologist did not think that the $2 \mathrm{AEs}$ were related to LA. AEs are discussed further in appendix e-2.

DISCUSSION This two-year, randomized, doubleblind, placebo-controlled pilot trial demonstrated a significant reduction in the primary outcome measure of the annualized rate of whole-brain atrophy by SIENA in people with SPMS taking $1,200 \mathrm{mg}$ daily oral LA. The annualized PCBV reduction was not influenced by the planned covariates of age, sex, and disease duration, nor by the post hoc additions of baseline brain and T2-lesion volumes. There was a suggestion of improved T25FW time, and there were fewer falls in the LA cohort. Overall LA was safe, well tolerated, had high compliance, and had no unexpected deleterious AEs or SAEs attributed to LA.

The reduction in the brain atrophy rate compares favorably with a large phase 3 trial of ocrelizumab $(\mathrm{n}=731)$, reporting a $17.5 \%$ reduction in the whole-brain atrophy rate over 120 weeks. ${ }^{26}$ In contrast to the present study, the ocrelizumab trial included only primary progressive MS patients with more men ( $51 \%$ vs $39 \%$ ) who were younger ( 45 vs 59 years), had shorter disease duration (6.5 vs 30 years), and less disability (EDSS 4.7 vs 5.4). The ocrelizumab trial limited inclusion to those with inflammatory CSF, a characteristic not assessed in this study. Other disease-modifying therapy trials in progressive MS populations have not demonstrated robust changes in brain atrophy rates or have not used this outcome measure. ${ }^{27-29}$

The PCBV reduction was for the whole-brain assessment by SIENA, a robust registration-based longitudinal atrophy measure. ${ }^{30}$ Brain segmentation did not reveal specific brain compartments 
responsible for differential atrophy rates. One explanation is that the sample size calculation was based on SIENA and not on segmentation methods, techniques which produce heterogeneous results and require larger samples. ${ }^{30}$ Segmented volumes may be more prone to physiologic factors including hydration status and positioning than whole-brain measurements. ${ }^{31}$ Alternatively, the PCBV reduction may come from an effect common across all brain tissues. Study replication in a larger sample will clarify the nature of LA's atrophy effects.

The suggestion of greater increase in T2-lesion volume in the LA cohort is of uncertain significance. The increase may be real and thus represent potential harmful effects of LA, may represent physiologic changes other than due to MS, may result from MR postprocessing quality issues, or may not be different from placebo. ${ }^{15}$ Further evaluation is warranted.

Overall, LA was safe and well tolerated. Laboratory abnormalities were limited to asymptomatic elevations in alkaline phosphatase which improved on cessation of LA. Although there were no unexpected AEs or SAEs clearly attributable to LA, the renal failure and glomerulonephritis cases raise concern. Previous studies with LA have not reported renal dysfunction..$^{9-11}$ Even so, closer renal function monitoring is prudent for future LA studies and suggests caution in recommendation of this dose of LA before further evaluations.

A potential study confounder of which the authors were unaware when planning the study was the presence of quercetin (8.6 mg daily) in the placebo. Quercetin, like other bioflavonoids, is biologically active. Although conflicting reports exist, Van Beek et al. found that $10 \mathrm{mg}$ daily oral quercetin increased inflammation and exacerbated active and passive EAE in mice. This raises the possibility that in the present study, quercetin could have worsened the controls and exaggerated the effects of LA. ${ }^{32}$ The calculated analogous human dose to the Van Beek study is $2 \mathrm{~g}$, orders of magnitude greater than the placebo capsules. ${ }^{33}$ Therefore, while unlikely to have affected present study outcomes, future studies should avoid quercetin to exclude this possibility.

Other study limitations relate primarily to the small sample size of this pilot study with resulting loss of power to detect clinical and secondary imaging outcomes as has been discussed. In light of this, the very robust reduction in the brain atrophy rate might be viewed with skepticism, although the comparable atrophy rate of the controls to other untreated SPMS cohorts lends credence to the findings. ${ }^{23,34}$ Baseline differences in brain volume or T2-lesion volume existed between study cohorts; however, post hoc analyses did not find an influence of these differences on study outcomes. A larger sample size in future studies should correct for baseline imbalances. Finally, as a contrast agent was not used, baseline and ongoing differences in inflammatory activity on MRI are unknown.

This 2-year pilot trial of 1,200 mg daily LA demonstrated significant reduction in PCBV in patients with SPMS over controls. The small sample size precluded detection of clinical benefits, although there was suggestion of improved walking times and significantly fewer falls. Although LA was overall safe and well tolerated, future studies need to establish clinical benefits and explore mechanisms of action of LA in progressive MS.

\section{AUTHOR CONTRIBUTIONS}

Rebecca Spain: study concept and design, study supervision, analysis and interpretation of data, drafting of manuscript, and obtained funding. Katherine Powers: data acquisition, MRI analyses, and drafting of manuscript. Charles Murchison: statistical analyses and drafting of the manuscript. Elizabeth Heriza: data acquisition and manuscript review. Kimberly Winges: review of OCTs, data analysis and interpretation of data, and manuscript review. Vijayshree Yadav, Michelle Cameron, and Edward Kim: data acquisition. Fay Horak: study concept and manuscript review. Jack Simon: study concept and design, analysis and interpretation of data, and drafting of manuscript. Dennis Bourdette: study concept and design, study supervision, analysis and interpretation of data, and critical review of the manuscript.

\section{ACKNOWLEDGMENT}

Pure Encapsulations, Sudbury, MA, provided the lipoic acid and placebo. The authors thank the study participants, Dr. Elizabeth Heriza for study management, and the members of the data safety and monitoring board for annual reviews of data.

\section{STUDY FUNDING}

Supported by the Department of Veterans Affairs (B7493-W, R. Spain), NIH (UL1TR000128).

\section{DISCLOSURE}

R. Spain received research support from Department of Veterans Affairs, Oregon Clinical and Translational Research Institute, VA Portland Health Care System, Oregon Health \& Science University, National MS Society, Conrad Hilton Foundation, Medical Research Foundation of Oregon, and Race to Erase MS. K. Powers, C. Murchison, E. Heriza, and K. Winges report no disclosures. V. Yadav consulted for Bayer, Biogen, and Teva MS; served on the speaker's bureau for Novartis and Biogen; and received research support from Biogen, Department of Veterans Affairs, McDougall Foundation, National MS Society, Race to Erase MS Foundation, and NIH. M. Cameron received travel funding from the Consortium of Multiple Sclerosis Centers and VA Portland Research; is an editorial board member for the Journal of Hand Therapy; section editor for Nature Reviews Neuroscience; receives publishing royalties from Elsevier; consulted for ReWalk Corporation and Adamas Corporation; received research support from the Department of Veterans Affairs, Rehabilitation Research and Development Service, National MS Society, and Paralyzed Veterans of America Foundation. E. Kim served on the scientific advisory board for Genzyme and Teva and received research support from the National MS Society. F. Horak served on the scientific advisory board for MS Society and NIH NCMRR; received travel funding and/or speaker honoraria for lectures and educational activities not funded by industry; served as an associate editor for Gait and Posture, Cerebellum, and Encyclopedia for Neuroscience; served on the editorial board for Journal of Biomechanics, Frontiers in Neurology, and Frontiers in Neuro-otology; holds patents for device for conditioning balance and motor coordination, Instrumented mobility system to 
objectively measure balance and gait; receives publishing royalties from McGraw-Hill; received research support from MRF NCI, NIH, SBIR, MRF, MRF Mentor, STTR, NIA, NCI, NIH/CCHD/NCMRR MJFF, NMSS, National Institute of Neurological Disorders and Stroke, NMSS Mentor, and US Army MRAA; and holds stock in APDM, Inc. J. Simon served on the scientific advisory board for Biogen; receives publishing royalties from Cambridge University Press; and consulted for Biogen, Guthy-Jackson Charitable Foundation. D. Bourdette received travel funding from National Multiple Sclerosis Society, Consortium of MS Centers, and Paralyzed Veterans of America; serves on the editorial board for Neurology; holds patents for treatment of multiple sclerosis with cyclic peptide derivatives of cyclosporin and thyromimetic drugs for stimulating remyelination in multiple sclerosis; consulted for Magellan Health, Best Doctors, Inc.; and received research support from National MS Society. Go to Neurology.org/nn for full disclosure forms.

Received March 7, 2017. Accepted in final form May 15, 2017.

\section{REFERENCES}

1. Mahad DH, Trapp BD, Lassmann H. Pathological mechanisms in progressive multiple sclerosis. Lancet Neurol 2015;14:183-193.

2. Furby J, Hayton T, Anderson V, et al. Magnetic resonance imaging measures of brain and spinal cord atrophy correlate with clinical impairment in secondary progressive multiple sclerosis. Mult Scler 2008;14:1068-1075.

3. Biewenga GP, Haenen GR, Bast A. The pharmacology of the antioxidant lipoic acid. Gen Pharmacol 1997;29: 315-331.

4. Biewenga GP, Dorstijn MA, Verhagen JV, Haenen GR, Bast A. Reduction of lipoic acid by lipoamide dehydrogenase. Biochem Pharmacol 1996;51:233-238.

5. Schreibelt G, Musters RJ, Reijerkerk A, et al. Lipoic acid affects cellular migration into the central nervous system and stabilizes blood-brain barrier integrity. J Immunol 2006;177:2630-2637.

6. Shay KP, Michels AJ, Li W, Kong AN, Hagen TM. Capindependent Nrf2 translation is part of a lipoic acidstimulated detoxification stress response. Biochim Biophys Acta 2012;1823:1102-1109.

7. Morini M, Roccatagliata L, Dell'Eva R, et al. Alpha-lipoic acid is effective in prevention and treatment of experimental autoimmune encephalomyelitis. J Neuroimmunol 2004;148:146-153.

8. Marracci GH, Jones RE, McKeon GP, Bourdette DN. Alpha lipoic acid inhibits $\mathrm{T}$ cell migration into the spinal cord and suppresses and treats experimental autoimmune encephalomyelitis. J Neuroimmunol 2002;131:104-114.

9. Yadav V, Marracci GH, Munar MY, et al. Pharmacokinetic study of lipoic acid in multiple sclerosis: comparing mice and human pharmacokinetic parameters. Mult Scler 2010;16:387-397.

10. Reljanovic M, Reichel G, Rett K, et al. Treatment of diabetic polyneuropathy with the antioxidant thioctic acid (alpha-lipoic acid): a two year multicenter randomized double-blind placebo-controlled trial (ALADIN II). Alpha Lipoic Acid in Diabetic Neuropathy. Free Radic Res 1999;31:171-179.

11. Yadav V, Marracci G, Lovera J, et al. Lipoic acid in multiple sclerosis: a pilot study. Mult Scler 2005;11:159-165.

12. Polman $\mathrm{CH}$, Reingold SC, Edan G, et al. Diagnostic criteria for multiple sclerosis: 2005 revisions to the “McDonald Criteria." Ann Neurol 2005;58:840-846.

13. Kurtzke JF. Rating neurologic impairment in multiple sclerosis: an expanded disability status scale (EDSS). Neurology 1983;33:1444-1452.
14. Schulz KF, Altman DG, Moher D, Group C. CONSORT 2010 statement: updated guidelines for reporting parallel group randomized trials. Ann Intern Med 2010; 152:726-732.

15. Shiee N, Bazin PL, Ozturk A, Reich DS, Calabresi PA, Pham DL. A topology-preserving approach to the segmentation of brain images with multiple sclerosis lesions. Neuroimage 2010;49:1524-1535.

16. Smith SM, Jenkinson M, Woolrich MW, et al. Advances in functional and structural MR image analysis and implementation as FSL. Neuroimage 2004;23(suppl 1): S208-S219.

17. Reuter M, Schmansky NJ, Rosas HD, Fischl B. Withinsubject template estimation for unbiased longitudinal image analysis. Neuroimage 2012;61:1402-1418.

18. Fischer JS, Rudick RA, Cutter GR, Reingold SC. The Multiple Sclerosis Functional Composite Measure (MSFC): an integrated approach to MS clinical outcome assessment. National MS Society Clinical Outcomes Assessment Task Force. Mult Scler 1999;5:244-250.

19. Powell LE, Myers AM. The Activities-specific Balance Confidence (ABC) Scale. J Gerontol A Biol Sci Med Sci 1995;50A:M28-M34.

20. Hobart JC, Riazi A, Lamping DL, Fitzpatrick R, Thompson AJ. Measuring the impact of MS on walking ability: the 12-Item MS Walking Scale (MSWS-12). Neurology 2003;60:31-36.

21. Parmenter BA, Weinstock-Guttman B, Garg N, Munschauer F, Benedict RH. Screening for cognitive impairment in multiple sclerosis using the Symbol digit Modalities Test. Mult Scler 2007;13:52-57.

22. RAND Corporation. 36-Item Short Form Survey (SF-36). Available at: rand.org/health/surveys_tools/mos/36-itemshort-form.html. Accessed May 2, 2017.

23. Altmann DR, Jasperse B, Barkhof F, et al. Sample sizes for brain atrophy outcomes in trials for secondary progressive multiple sclerosis. Neurology 2009;72:595-601.

24. R Core Team. R: A Language and Environment for Statistical Computing. Vienna: R Foundation for Statistical Computing [Internet]; 2016. Available at: R-project.org/. Accessed May 2, 2017.

25. Bates D, Mächler M, Bolker B, Walker S. Fitting linear mixed-effects models using lme4. J Stat Softw 2015;67:1-48.

26. Montalban X, Hemmer B, Rammohan K, et al. Efficacy and safety of ocrelizumab in primary progressive multiple sclerosis-results of the placebo-controlled, double-blind, Phase III ORATORIO study. Barcelona, Spain: ECTRIMS Online Library; 2015.

27. Kapoor R, Furby J, Hayton T, et al. Lamotrigine for neuroprotection in secondary progressive multiple sclerosis: a randomised, double-blind, placebo-controlled, parallel-group trial. Lancet Neurol 2010;9:681-688.

28. Chataway J, Schuerer N, Alsanousi A, et al. Effect of high-dose simvastatin on brain atrophy and disability in secondary progressive multiple sclerosis (MS-STAT): a randomised, placebo-controlled, phase 2 trial. Lancet 2014;383:2213-2221.

29. Tourbah A, Lebrun-Frenay C, Edan G, et al. MD1003 (high-dose biotin) for the treatment of progressive multiple sclerosis: a randomised, double-blind, placebo-controlled study. Mult Scler 2016;22:1719-1731.

30. Durand-Dubief F, Belaroussi B, Armspach JP, et al. Reliability of longitudinal brain volume loss measurements between 2 sites in patients with multiple sclerosis: 
comparison of 7 quantification techniques. AJNR Am J Neuroradiol 2012;33:1918-1924.

31. Sampat MP, Healy BC, Meier DS, Dell'Oglio E, Liguori M, Guttmann CR. Disease modeling in multiple sclerosis: assessment and quantification of sources of variability in brain parenchymal fraction measurements. Neuroimage 2010;52:1367-1373.

32. Verbeek R, van Tol EA, van Noort JM. Oral flavonoids delay recovery from experimental autoimmune encephalomyelitis in SJL mice. Biochem Pharmacol 2005;70:220-228.

33. Nair AB, Jacob S. A simple practice guide for dose conversion between animals and human. J Basic Clin Pharm 2016;7:27-31.

34. De Stefano N, Giorgio A, Battaglini M, et al. Assessing brain atrophy rates in a large population of untreated multiple sclerosis subtypes. Neurology 2010;74:18681876. 


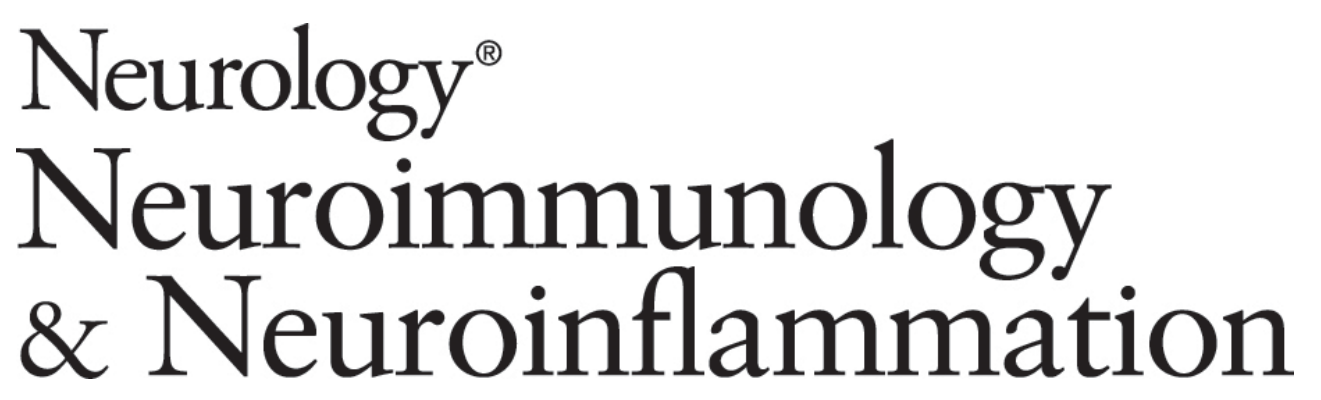

Lipoic acid in secondary progressive MS: A randomized controlled pilot trial Rebecca Spain, Katherine Powers, Charles Murchison, et al.

Neurol Neuroimmunol Neuroinflamm 2017;4;

DOI 10.1212/NXI.0000000000000374

This information is current as of June 28, 2017

Neurol Neuroimmunol Neuroinflamm is an official journal of the American Academy of Neurology.

Published since April 2014, it is an open-access, online-only, continuous publication journal. Copyright

Copyright $\odot 2017$ The Author(s). Published by Wolters Kluwer Health, Inc. on behalf of the American

Academy of Neurology.. All rights reserved. Online ISSN: 2332-7812.

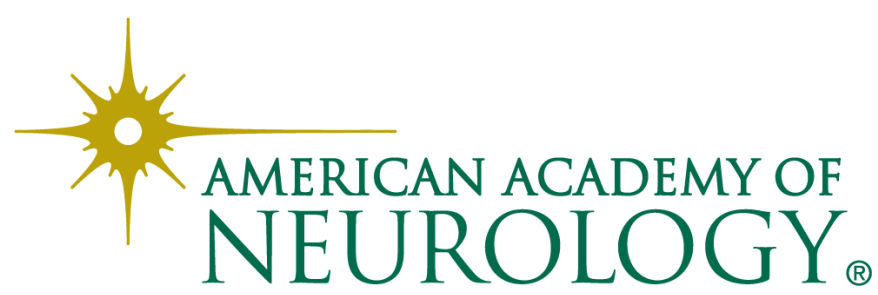




\section{Updated Information \& Services}

\section{Supplementary Material}

\section{References}

Citations

Subspecialty Collections

\section{Permissions \& Licensing}

Reprints including high resolution figures, can be found at: http://nn.neurology.org/content/4/5/e374.full.html

Supplementary material can be found at: http://nn.neurology.org/content/suppl/2017/07/20/4.5.e374.DC1

This article cites 31 articles, 2 of which you can access for free at: http://nn.neurology.org/content/4/5/e374.full.html\#\#ref-list-1

This article has been cited by 7 HighWire-hosted articles: http://nn.neurology.org/content/4/5/e374.full.html\#\#otherarticles

This article, along with others on similar topics, appears in the following collection(s):

\section{Class I}

http://nn.neurology.org//cgi/collection/class_1

Clinical trials Randomized controlled (CONSORT agreement)

http://nn.neurology.org//cgi/collection/clinical_trials_randomized_cont rolled_consort_agreement

Multiple sclerosis

http://nn.neurology.org//cgi/collection/multiple_sclerosis

\section{Volumetric MRI}

http://nn.neurology.org//cgi/collection/volumetric_mri

Information about reproducing this article in parts (figures,tables) or in its entirety can be found online at:

http://nn.neurology.org/misc/about.xhtml\#permissions

Information about ordering reprints can be found online: http://nn.neurology.org/misc/addir.xhtml\#reprintsus

Neurol Neuroimmunol Neuroinflamm is an official journal of the American Academy of Neurology.

Published since April 2014, it is an open-access, online-only, continuous publication journal. Copyright

Copyright $\odot 2017$ The Author(s). Published by Wolters Kluwer Health, Inc. on behalf of the American Academy of Neurology.. All rights reserved. Online ISSN: 2332-7812.

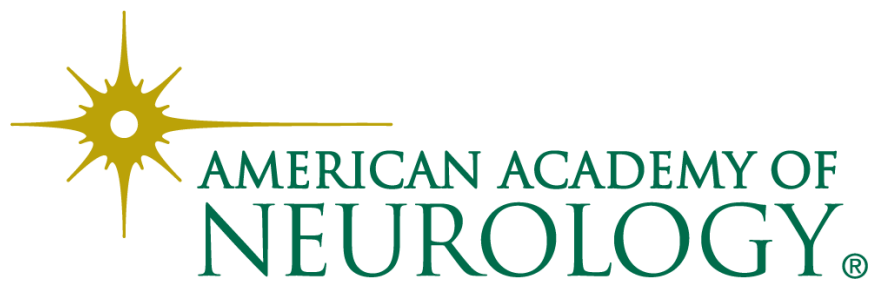

